\title{
Methanol extract of Sanguisorba officinalis L. with cytotoxic activity against PC3 human prostate cancer cells
}

\author{
EUN-SUN CHOI ${ }^{1 *}$, JUN-SUNG KIM ${ }^{2 *}$, KI-HAN KWON ${ }^{3}$, HYNG-SEOP KIM $^{4}$, \\ NAM-PYO $\mathrm{CHO}^{1}$ and SUNG-DAE $\mathrm{CHO}^{1}$
}

\begin{abstract}
${ }^{1}$ Department of Oral Pathology, School of Dentistry, Institute of Oral Bioscience, Chonbuk National University, Jeonju 561-756; ${ }^{2}$ \&\&D Center, Biterials Co. Ltd., Seoul 140-200; ${ }^{3}$ Department of Food Science and Nutrition, College of Health, Welfare and Education, Gwangju University, Gwangju 503-703; ${ }^{4}$ Department of Oral Periodontology, School of Dentistry, Institute of Oral Bioscience, Chonbuk National University, Jeonju 561-756, Republic of Korea
\end{abstract}

Received April 6, 2012; Accepted June 12, 2012

DOI: $10.3892 / \mathrm{mmr} .2012 .949$

\begin{abstract}
Sanguisorba officinalis is a natural plant that has been traditionally used for the treatment of inflammatory and metabolic diseases. Several studies have reported that its extracts exhibit anticancer, antioxidative and anti-lipid peroxidation activities. However, the effects of this plant on human prostate cancer cells have not yet been investigated. In the present study, we investigated the inhibitory effects and underlying mechanisms of a methanol extract of Sanguisorba officinalis (MESO) in PC3 human prostate cancer cells. MESO significantly decreased cell growth and induced apoptosis through the intrinsic apoptosis pathway. MESO decreased the expression levels of myeloid cell leukemia-1 (Mcl-1), a Bcl-2-like anti-apoptotic protein that is highly expressed in various cancer cell lines. Expression levels of the pro-apoptotic protein Bax were increased by MESO whereas those of Bak and $\mathrm{Bcl}-\mathrm{xL}$ were unchanged. In addition, MESO induced the oligomerization of Bax in the mitochondrial outer membrane. These results suggest that MESO inhibits the growth of prostate cancer cells and induces apoptotic cell death by the downregulation of Mcl-1 protein expression and the oligomerization of Bax. Therefore, MESO has potential as a drug candidate for the treatment of prostate cancer.
\end{abstract}

\section{Introduction}

Prostate cancer is the most common form of cancer among men in the US. In 2010, it was reported that 220,000 men

Correspondence to: Dr Sung-Dae Cho, Department of Oral Pathology, School of Dentistry, Institute of Oral Bioscience, Chonbuk National University, Jeonju 561-756, Republic of Korea E-mail: efiwdsc@chonbuk.ac.kr

\section{*Contributed equally}

Key words: Sanguisorba officinalis L., myeloid cell leukemia-1, Bax, oligomerization, prostate cancer were newly diagnosed with prostate cancer and 32,050 men succumbed to the disease (1). Almost one in six men are likely to develop prostate cancer at some point in their lives, with the majority of incidences occurring after the age of 50 (2). Various therapies, including hormones, surgery, radiation and chemotherapy, have been used for the treatment of prostate cancer. However, all of these treatments suffer from limitations, and in the majority of cases, a relapse of the disease occurs (3). Therefore, new strategies for the treatment of prostate cancer are required (4).

The Bcl-2 family of proteins, including anti-apoptotic [Bcl-2, Bcl-xL and myeloid cell leukemia-1 (Mcl-1)] and pro-apoptotic (Bax, Bak, Bad, Bid, Bim, Puma and Noxa) members, regulate apoptotic processes through an intrinsic mitochondrial apoptosis signaling pathway (5). One of the anti-apoptotic proteins, Mcl-1, is highly expressed in a number of cancer cell types to promote their survival (5-8). Several studies have revealed that Mcl-1 expression correlates with the resistance of cancer cells to chemotherapy and that the genetic inhibition of Mcl-1 induces apoptosis in several types of cancer (9-13). Interestingly, recent studies have shown that the overexpression of Mcl-1 in cancer cells is inhibited by various medicinal plants to induce apoptosis $(9,14,15)$.

Sanguisorba officinalis L. is a valuable medicinal plant in Korea, China and Japan, where it is used traditionally for the treatment of inflammatory and metabolic diseases, including diarrhea, chronic intestinal infections, duodenal ulcers and bleeding (16). Previous studies have reported that $S$. officinalis L. includes various biologically active compounds and exhibits anticancer activity $(17,18)$. However, the molecular mechanism underlying this anticancer activity has not yet been fully investigated. Therefore, the aims of this study were to evaluate the growth-inhibitory effect of a methanol extract of S. officinalis L. (MESO) on human prostate cancer cells and to elucidate the signaling pathway instrumental in mediating MESO-induced apoptosis in human prostate cancer cells. This study provides evidence that MESO induces apoptotic cell death to inhibit the growth of prostate cancer cells. In addition, the downregulation of Mcl-1 expression and the oligomerization of Bax in the mitochondrial outer membrane are revealed to mediate the apoptotic cell death. 


\section{Materials and methods}

Reagents. The antibodies to cleaved caspase 3, Mcl-1, Bax, Bak and Bcl-xL were purchased from Cell Signaling Technology, Inc. (Danvers, MA, USA). Actin antibody was purchased from Santa Cruz Biotechnology, Inc. (Santa Cruz, CA, USA). 4',6-Diamidino-2-phenylindole (DAPI) and propidium iodide (PI) were acquired from Sigma-Aldrich Chemical Co. (St. Louis, MO, USA). MESO was obtained from Professor K.H. Kwon (Gwangju, Korea).

Cell culture and chemical treatment. The PC3 human prostate cancer cells were provided by Dr Hwan-Mook Kim (Korea Research Institute of Bioscience and Biotechnology, Daejeon, Korea). The cells were cultured in RPMI medium containing $10 \%$ fetal bovine serum (FBS) and $100 \mathrm{U} / \mathrm{ml}$ each of penicillin and streptomycin (WelGENE Inc., Daegu, South Korea) in a humid atmosphere of $5 \% \mathrm{CO}_{2}$. Equal numbers of cells were seeded and allowed to attach overnight. The cells were treated with $0.1 \%$ dimethyl sulfoxide (DMSO) or MESO (40, 80 and $120 \mu \mathrm{g} / \mathrm{ml}$ ) diluted in RPMI with 5\% FBS for $48 \mathrm{~h}$.

MTS assay. We used the CellTiter 96 Aqueous One Solution Cell Proliferation Assay kit (Promega Corporation, Madison, WI, USA) for the estimation of cell viability. The cells were seeded in 96-well plates and incubated with various concentrations of MESO. Following treatment with MESO for $48 \mathrm{~h}$, $30 \mu 1$ MTS [3-(4,5-dimethylthiazol-2-yl)-5-(3-carboxymethoxyphenyl)-2-(4-sulfo-phenyl)-2H-tetrazolium] solution was added to each well and the cells were incubated for $2 \mathrm{~h}$ at $37^{\circ} \mathrm{C}$. The MTS solution was analyzed using a microplate reader (BioTek Instruments, Inc., Winooski, VT, USA) at 490 and $690 \mathrm{~nm}$ (background).

DAPI staining. DAPI staining was performed to determine the morphology of the cell nuclei following treatment with MESO. Briefly, the PC3 cells were treated with MESO or $0.1 \%$ DMSO and harvested by trypsinization. The cells were resuspended in PBS, deposited on poly-L-lysine-coated slides, stained with DAPI solution $(2 \mu \mathrm{g} / \mathrm{ml})$ and observed under a fluorescence microscope.

Western blot analysis. The MESO-treated PC3 cells were harvested and suspended in lysis buffer. Following sonication, the cell lysates were cleared by centrifugation at 13,000 rpm for $20 \mathrm{~min}$ at $4^{\circ} \mathrm{C}$. The protein supernatant fractions were subjected to SDS-PAGE and then transferred to polyvinylidene difluoride (PVDF) membranes and blocked with 5\% skimmed milk in Tris-buffered saline containing $0.1 \%$ Tween 20 (TBST). The PVDF membranes were incubated with primary antibody in TBST overnight at $4^{\circ} \mathrm{C}$. The membranes were then washed with TBST and incubated with secondary antibody in $5 \%$ skimmed milk in TBST for $90 \mathrm{~min}$ at room temperature (RT). After washing with TBST, the membranes were developed using an enhanced chemiluminescence detection kit (ECL, Santa Cruz Biotechnology).

Crosslinking. To evaluate Bax oligomerization, the PC3 cells were treated with DMSO or MESO for $48 \mathrm{~h}$. The cells were harvested and suspended in a conjugation buffer with $10 \mathrm{mM}$
EDTA. The lysates were incubated with $0.2 \mathrm{mM}$ 1,6-bismaleimidohexane (BMH, Thermo Fisher Scientific, Waltham, MA, USA) at RT for $1 \mathrm{~h}$ and then extracted using lysis buffer for western blot analysis.

Statistical analysis. Data were assessed for statistical significance using the Student's t-test. A p-value $<0.05$ compared with the vehicle control was considered to indicate a statistically significant result.

\section{Results}

MESO decreases the growth of PC3 cells. To determine the growth inhibitory effect of MESO in PC3 cells, we first investigated the morphological changes in the cells using optical microscopy. The images revealed that the cells rounded up and their numbers clearly decreased in a concentration-dependent manner (Fig. 1A). The effect of MESO on cell viability was examined using an MTS assay. MESO inhibited the proliferation of the PC3 cells in a concentration-dependent manner. The $\mathrm{ID}_{50}$ value of MESO for the PC3 cells was $120 \mu \mathrm{g} / \mathrm{ml}$ (Fig. 1B). These results suggest that MESO is an inhibitor of PC3 human prostate cancer cell growth.

MESO induces apoptosis through an intrinsic signaling pathway in PC3 cells. To investigate whether the MESO-induced growth inhibition was related to an apoptotic effect, apoptotic cell death in the MESO-treated PC3 cells was evaluated by DAPI staining and western blot analysis using anti-caspase 9 and anti-caspase 3 . The results revealed that the treatment of the cells with MESO increased the number of condensed and fragmented nuclei compared with DMSO treatment (Fig. 2A). In addition, MESO activated caspase 9 and caspase 3 (Fig. 2B). These results suggest that the growth inhibitory effect of MESO in the PC3 cells was due to apoptotic cell death.

MESO increases the level of Bax expression by inhibiting the Mcl-1 anti-apoptotic protein. Having confirmed that MESO induced apoptosis and thereby inhibited the growth of PC3 cells, we next investigated the molecular mechanism underlying the MESO-induced apoptosis. When the PC3 cells were exposed to MESO for $48 \mathrm{~h}$, the expression levels of Mcl-1 protein decreased in a concentration-dependent manner (Fig. 3A). We also analyzed the expression levels of the Bcl-2 family proteins that are essential for apoptotic signaling. The results demonstrated that the expression levels of Bax protein in the PC3 cells were increased by MESO, whereas those of Bak and Bcl-xL proteins were not (Fig. 3B). This suggests that MESO reduces the $\mathrm{Mcl}-1$ protein levels and increases the Bax protein levels in PC3 cells to induce apoptosis.

MESO increases Bax oligomerization in the mitochondrial outer membrane. According to previous studies, when apoptotic signals are received, $\mathrm{BH} 3$-only proteins competitively bind to the hydrophobic groove of the anti-apoptotic proteins and displace Bax, which mediates structural reorganization, leading to mitochondrial targeting and homo-oligomerization (19). Therefore, we examined whether MESO affected Bax oligomerization in the PC3 cells. The results revealed that MESO increased Bax oligomerization in a concentration- 
A
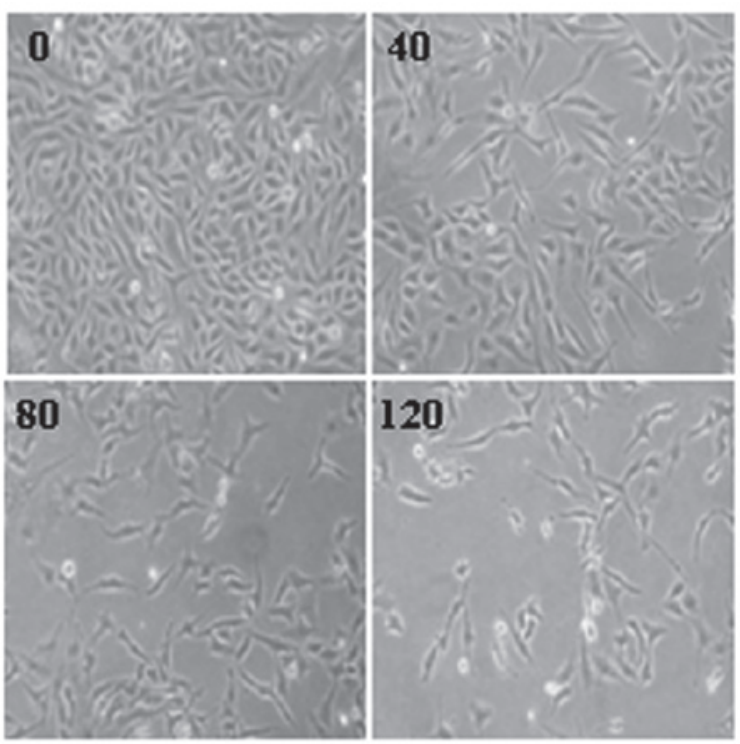

B

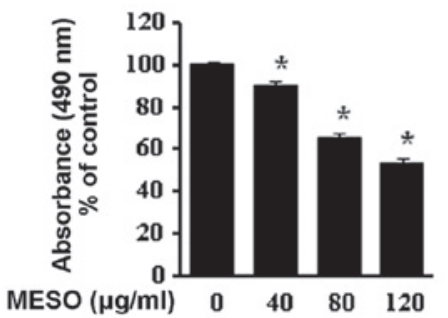

Figure 1. The effect of MESO on PC3 cell growth. (A) Photomicrographs (magnification, x100), captured with a light microscope, of PC3 cells treated with DMSO (vehicle control) or with 40,80 or $120 \mu \mathrm{g} / \mathrm{ml}$ MESO for $48 \mathrm{~h}$. (B) The viability of PC3 cells treated with DMSO or with 40, 80 or $120 \mu \mathrm{g} / \mathrm{ml}$ of MESO for $48 \mathrm{~h}$ determined by MTS assay. "p $<0.05 \mathrm{com}-$ pared with the DMSO-treated cells. MESO, methanol extract of Sanguisorba officinalis; DMSO, dimethyl sulfoxide; MTS, 3-(4,5-dimethylthiazol-2-yl)5-(3-carboxymethoxyphenyl)-2-(4-sulfophenyl)-2H-tetrazolium.

dependent manner (Fig. 4). This suggests that MESO promotes Bax oligomerization in the mitochondrial outer membranes of the PC3 cells.

\section{Discussion}

Several studies have revealed that certain naturally occurring medicinal plants inhibit the growth of various cancers (20-22). Specifically, it has been reported that the use of medicinal plants among prostate cancer patients is extremely popular $(23,24)$. One of these plants, Sanguisorba officinalis L., has been effectively used for the treatment of inflammation and metabolic diseases, as well as cancer $(24,25)$. Its ethanol extract exhibits anticancer activity by inhibiting nitric oxide (NO) and prostaglandin E2 through suppression of the NF- $\mathrm{KB}$ and AP-1 activation signaling cascades. However, the anticancer activity of a methanol extract in PC3 human prostate cancer cells has not yet been investigated. Therefore, we evaluated the effects of MESO on the growth of PC3 cells and the mechanisms underlying these effects.

Initially, we investigated the effects of MESO on cell morphology and viability using light microscopic observation and MTS assays. The exposure of the PC3 cells to various concentrations of MESO clearly caused a
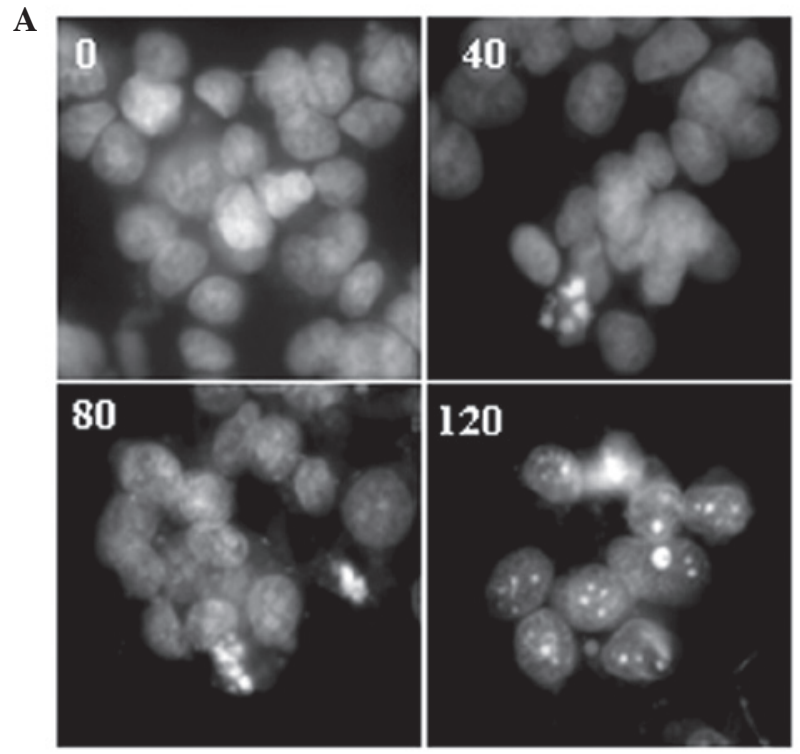

B

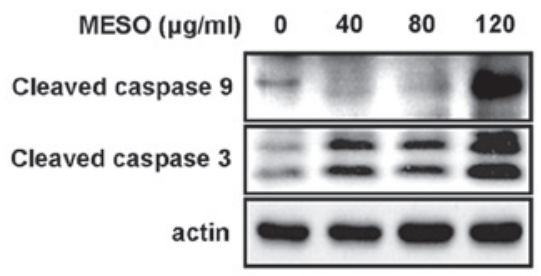

Figure 2. The apoptotic effect of MESO on PC3 cells. (A) PC3 cells treated with DMSO or with 40,80 or $120 \mu \mathrm{g} / \mathrm{ml}$ MESO for $48 \mathrm{~h}$, determined by DAPI staining. Fluorescence microscopy (magnification x400) images reveal nuclear condensation and fragmentation in the MESO-treated PC3 cells. (B) Expression levels of cleaved caspase 9 and caspase 3 in whole cell lysates from PC 3 cells treated with MESO for $48 \mathrm{~h}$ were detected by western blot analysis. Actin was used as a loading control to normalize the protein levels. MESO, methanol extract of Sanguisorba officinalis; DMSO, dimethyl sulfoxide; DAPI, 4',6-diamidino-2-phenylindole.

A

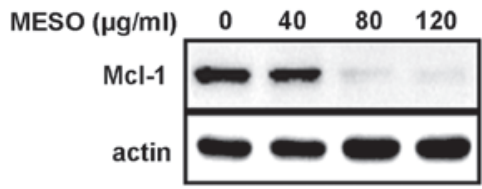

B

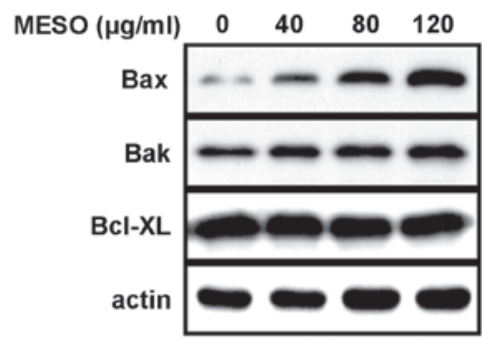

Figure 3. The effect of MESO on Bcl-2 family proteins. The expression levels of (A) Mcl-1 and (B) Bcl-2 family proteins (Bax, Bak and Bcl-xL) were analyzed by western blot analysis. MESO, methanol extract of Sanguisorba officinalis; Mcl-1, myeloid cell leukemia-1.

concentration-dependent inhibition of cell growth and cell detachment, suggesting that MESO inhibited the proliferation of the prostate cancer cells by affecting cell viability. We then 


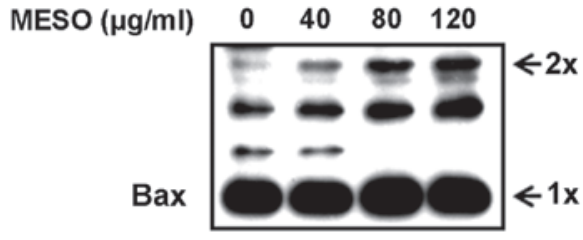

Figure 4. The effect of MESO on Bax oligomerization in the mitochondrial outer membrane. The cell lysates from MESO-treated PC3 cells were treated with $0.2 \mathrm{mM} \mathrm{BMH}$ and analyzed by western blot analysis with anti-Bax MESO, methanol extract of Sanguisorba officinalis; BMH, 1,6-bismaleimidohexane.

investigated the apoptotic effects of MESO in the PC3 cells and found that MESO induced apoptosis, as evidenced by the concentration-dependent appearance of nuclear condensation and fragmentation and increased amounts of cleaved poly(ADP-ribose) polymerase. Mammalian cell apoptosis is initiated by intrinsic or extrinsic pathways (20). The intrinsically mediated pathway is known as the mitochondria-initiated pathway and in this pathway cytochrome $\mathrm{c}$ is released from the mitochondria, which converts procaspase 9 into active caspase 9. Activated caspase 9 then cleaves and activates downstream caspases, including caspases 3, 6 and 7 (19). We sought to determine whether the MESO-induced apoptosis was intrinsic or extrinsic. The results revealed that the levels of the cleaved forms of caspase 9 and caspase 3 were increased by MESO, indicating that MESO induced apoptosis through the intrinsic signaling pathway to inhibit the growth of PC3 cells.

Numerous apoptosis-related genes, including pro-apoptotic genes (Bax and Bak) and anti-apoptotic genes (Bcl-xL, Bcl-2 and $\mathrm{Mcl}-1$ ), play significant roles in the apoptotic signaling pathway. The expression of Mcl-1 protects cancer cells from the apoptotic signaling pathway (26). Several studies have reported that prostate carcinogenesis is induced by the involvement of overexpressed Mcl-1 genes, and that downregulation of the Mcl-1 gene leads to apoptosis in prostate cancer cells $(27,28)$. This suggests that Mcl-1 is a good molecular target for the treatment of prostate cancer. Since the mechanism by which MESO exerts its apoptotic effects is unclear, we investigated the effects of MESO on the expression of the Mcl-1 protein in PC3 cells. We found that MESO decreased the expression levels of the Mcl-1 protein. The Mcl-1 protein is primarily localized in the outer mitochondrial membrane and promotes cell survival by suppressing cytochrome c release from the mitochondria via hetero-dimerization with, and neutralization of, effector pro-apoptotic Bcl-2 family members, including Bax and Bak $(5,29,30)$. However, when apoptotic signals are received, activator $\mathrm{BH} 3$-only proteins (Bim, PUMA and tBid) bind and activate Bax and/or Bak directly if they are not bound and neutralized by Bcl-2-like proteins, including Mcl-1 (5,31-36). Thus, we investigated the effects of MESO on Bax, Bak and Bcl-XL. The data demonstrated that MESO increased the Bax protein levels in PC3 cells whereas the levels of Bak and Bcl-xL proteins were unchanged. These findings indicated that MESO may regulate Bax protein levels as a downstream molecule of the Mcl-1 protein.

The permeabilization of the mitochondrial outer membrane is initiated by changes in the expression of $\mathrm{Bcl}-2$ family proteins. The structural reorganization of Bax from its inactive conformation leads to mitochondrial targeting and homo-oligomerization (19). Oligomerization releases cytochrome $\mathrm{c}$ from the mitochondrial intermembrane space into the cytosol, where it binds to Apaf-1 and coordinates the formation of the Apaf-1/caspase 9 apoptosome (37-39). Bax activation is mediated by structural reorganization and leads to mitochondrial targeting and homo-oligomerization (19). Several studies have reported that various anticancer drugs derived from plant extracts induce apoptosis in cancer cells that is accompanied by Bax oligomerization and the release of cytochrome $\mathrm{c}$ from the mitochondria into the cytosol (40-42). Therefore, we sought to confirm the occurrence of Bax oligomerization in the PC-3 cells. In our study, we revealed that MESO induced Bax oligomerization in the PC3 cells in a concentration-dependent manner. These findings suggest that the MESO-induced Bax oligomerization promotes apoptosis through an intrinsic mitochondria-initiated apoptosis signaling pathway in the PC3 human prostate cancer cells.

In conclusion, we demonstrated that MESO has a growth inhibitory effect on PC3 cells and induces apoptosis via an intrinsic apoptotic pathway. We also provided evidence that the apoptotic effect of MESO is caused by the modulation of Mcl-1 and Bax protein levels, leading to the oligomerization of Bax in the mitochondrial outer membranes. Therefore, we suggest that MESO is a meaningful medicinal plant extract and a drug candidate for the treatment of prostate cancer.

\section{Acknowledgements}

This study is supported by National Research Foundation of Korea (NRF) funded by the Ministry of Education, Science, and Technology [20111-0019173].

\section{References}

1. Dayyani F, Gallick GE, Logothetis CJ and Corn PG: Novel therapies for metastatic castrate-resistant prostate cancer (Review). J Natl Cancer Inst 103: 1665-1675, 2011.

2. Eisermann K, Tandon S, Bazarov A, Brett A, Fraizer G and Piontkivska $\mathrm{H}$ : Evolutionary conservation of zinc finger transcription factor binding sites in promoters of genes co-expressed with WT1 in prostate cancer. BMC Genomics 9: 337, 2008.

3. Kaur M and Agarwal R: Transcription factors: molecular targets for prostate cancer intervention by phytochemicals (Review). Curr Cancer Drug Targets 7: 355-367, 2007.

4. Bardia A, Platz EA, Yegnasubramanian S, De Marzo AM and Nelson WG: Anti-inflammatory drugs, antioxidants, and prostate cancer prevention (Review). Curr Opin Pharmacol 9: 419-426, 2009.

5. Akgul C: Mcl-1 is a potential therapeutic target in multiple types of cancer (Review). Cell Mol Life Sci 66: 1326-1336, 2009.

6. Tang H, Shao H, Yu C and Hou J: Mcl-1 downregulation by YM155 contributes to its synergistic anti-tumor activities with ABT-263. Biochem Pharmacol 82: 1066-1072, 2011.

7. Zhang Z, Yang H, Wu G, Li Z, Song T and Li XQ: Probing the difference between $\mathrm{BH} 3$ groove of $\mathrm{Mcl}-1$ and $\mathrm{Bcl}-2$ protein: Implications for dual inhibitors design. Eur J Med Chem 46: 3909-3916, 2011.

8. Warr MR and Shore GC: Unique biology of Mcl-1: therapeutic opportunities in cancer (Review). Curr Mol Med 8: 138-147, 2008.

9. Takahashi H, Chen MC, Pham H, et al: Baicalein, a component of Scutellaria baicalensis, induces apoptosis by Mcl-1 downregulation in human pancreatic cancer cells. Biochim Biophys Acta 1813: 1465-1474, 2011.

10. Chen W, Bai L, Wang X, Xu S, Belinsky SA and Lin Y: Acquired activation of the Akt/cyclooxygenase-2/Mcl-1 pathway renders lung cancer cells resistant to apoptosis. Mol Pharmacol 77: 416-423, 2010. 
11. Wei SH, Dong K, Lin F, et al: Inducing apoptosis and enhancing chemosensitivity to gemcitabine via RNA interference targeting Mcl-1 gene in pancreatic carcinoma cell. Cancer Chemother Pharmacol 62: 1055-1064, 2008.

12. Schulze-Bergkamen H, Fleischer B, Schuchmann M, et al: Suppression of Mcl-1 via RNA interference sensitizes human hepatocellular carcinoma cells towards apoptosis induction. BMC Cancer 6: 232, 2006.

13. Guoan X, Hanning W, Kaiyun C and Hao L: Adenovirusmediated siRNA targeting Mcl-1 gene increases radiosensitivity of pancreatic carcinoma cells in vitro and in vivo. Surgery 147: 553-561, 2010.

14. Bredholt T, Dimba EA, Hagland HR, et al: Camptothecin and khat (Catha edulis Forsk.) induced distinct cell death phenotypes involving modulation of c-FLIPL, Mcl-1, procaspase- 8 and mitochondrial function in acute myeloid leukemia cell lines. Mol Cancer 8: 101, 2009.

15. Kim YI, Park SW, Choi IH, Lee JH, Woo HJ and Kim Y: Effect of Orostachys japonicus on cell growth and apoptosis in human hepatic stellate cell line LX2. Am J Chin Med 39: 601-613, 2011.

16. Lee NH, Lee MY, Lee JA, et al: Anti-asthmatic effect of Sanguisorba officinalis L. and potential role of heme oxygenase-1 in an ovalbumin-induced murine asthma model. Int $\mathrm{J}$ Mol Med 26: 201-208, 2010.

17. Cho JY, Yoo ES, Cha BC, Park HJ, Rhee MH and Han YN: The inhibitory effect of triterpenoid glycosides originating from Sanguisorba officinalis on tissue factor activity and the production of TNF-alpha. Planta Med 72: 1279-1284, 2006.

18. Goun EA, Petrichenko VM, Solodnikov SU, et al: Anticancer and antithrombin activity of Russian plants. J Ethnopharmacol 81: 337-342, 2002.

19. Ma Y, Zhang A, Shi Z, et al: A mitochondria-mediated apoptotic pathway induced by deoxynivalenol in human colon cancer cells. Toxicol In Vitro 26: 414-420, 2012.

20. Wang X, Zhang F, Yang L, et al: Ursolic acid inhibits proliferation and induces apoptosis of cancer cells in vitro and in vivo. J Biomed Biotechnol 2011: 419343, 2011.

21. Chen XR, Lu R, Dan HX, et al: Honokiol: a promising small molecular weight natural agent for the growth inhibition of ora squamous cell carcinoma cells. Int J Oral Sci 3: 34-42, 2011.

22. Wang Y, Deng L, Zhong H, Jiang X and Chen J: Natural plant extract tubeimoside I promotes apoptosis-mediated cell death in cultured human hepatoma (HepG2) cells. Biol Pharm Bull 34: 831-838, 2011.

23. Chun JY, Tummala R, Nadiminty N, et al: Andrographolide, a herbal medicine, inhibits interleukin- 6 expression and suppresses prostate cancer cell growth. Genes Cancer 1: 868-876, 2010.

24. Lin YH, Chen KK and Chiu JH: Coprescription of Chinese herbal medicine and western medications among prostate cancer patients: a population-based study in Taiwan. Evid Based Complement Alternat Med 2012: 147015, 2012.

25. Wang Z, Loo WT, Wang N, Chow LW, Wang D, Han F, Zheng X and Chen JP: Effect of Sanguisorba officinalis L. on breast cancer growth and angiogenesis. Expert Opin Ther Targets 16 (Suppl 1): S79-S89, 2012.

26. Quinn BA, Dash R, Azab B, et al: Targeting Mcl-1 for the therapy of cancer (Review). Expert Opin Investig Drugs 20: 1397-1411, 2011.
27. Dash R, Azab B, Quinn BA, et al: Apogossypol derivative BI-97C1 (Sabutoclax) targeting Mcl-1 sensitizes prostate cancer cells to mda-7/IL-24-mediated toxicity. Proc Natl Acad Sci USA 108: 8785-8790, 2011

28. Senft D, Berking C, Graf SA, Kammerbauer C, Ruzicka T and Besch R: Selective induction of cell death in melanoma cell lines through targeting of Mcl-1 and A1. PLoS One 7: e30821, 2012.

29. Shimazu T, Degenhardt K, Nur-E-Kamal A, et al: NBK/BIK antagonizes MCL-1 and BCL-XL and activates BAK-mediated apoptosis in response to protein synthesis inhibition. Genes Dev 21: 929-941, 2007.

30. Dewson G and Kluck RM: Mechanisms by which Bak and Bax permeabilise mitochondria during apoptosis. J Cell Sci 122: 2801-2808, 2009.

31. Kim H, Rafiuddin-Shah M, Tu HC, et al: Hierarchical regulation of mitochondrion-dependent apoptosis by BCL-2 subfamilies. Nat Cell Biol 8: 1348-1358, 2006.

32. Clohessy JG, Zhuang J, de Boer J, Gil-Gómez G and Brady HJ: Mcl-1 interacts with truncated Bid and inhibits its induction of cytochrome c release and its role in receptor-mediated apoptosis. J Biol Chem 281: 5750-5759, 2006.

33. Adams JM and Cory S: Bcl-2-regulated apoptosis: mechanism and therapeutic potential (Review). Curr Opin Immunol 19: 488-496, 2007.

34. Mitchell C, Yacoub A, Hossein H, et al: Inhibition of MCL-1 in breast cancer cells promotes cell death in vitro and in vivo. Cancer Biol Ther 10: 903-917, 2010.

35. Ménoret E, Gomez-Bougie P, Surget S, et al: Mcl-1(128-350) fragment induces apoptosis through direct interaction with Bax. FEBS Lett 584: 487-492, 2010.

36. Jiang CC, Wroblewski D, Yang F, Hersey P and Zhang XD: Human melanoma cells under endoplasmic reticulum stress are more susceptible to apoptosis induced by the $\mathrm{BH} 3$ mimetic obatoclax. Neoplasia 11: 945-955, 2009.

37. van Delft MF and Huang DC: How the Bcl-2 family of proteins interact to regulate apoptosis (Review). Cell Res 16: 203-213, 2006.

38. Adams JM: Ways of dying: multiple pathways to apoptosis. Genes Dev 17: 2481-2495, 2003.

39. Green DR and Kroemer G: The pathophysiology of mitochondrial cell death (Review). Science 305: 626-629, 2004.

40. Chu R, Upreti M, Ding WX, Yin XM and Chambers TC: Regulation of Bax by c-Jun NH2-terminal kinase and Bcl-xL in vinblastine-induced apoptosis. Biochem Pharmacol 78: 241-248, 2009.

41. Qanungo S, Das M, Haldar S and Basu A: Epigallocatechin3-gallate induces mitochondrial membrane depolarization and caspase-dependent apoptosis in pancreatic cancer cells. Carcinogenesis 26: 958-967, 2005.

42. Zhao L, He F, Liu H, et al: Natural diterpenoid compound elevates expression of Bim protein, which interacts with antiapoptotic protein $\mathrm{Bcl}-2$, converting it to proapoptotic Bax-like molecule. J Biol Chem 287: 1054-1065, 2012. 